\title{
WT ASP
} $\downarrow$

Polarized expression of the membrane ASP protein derived from HIV-1 antisense transcription in T cells

Clerc et al. 


\title{
Polarized expression of the membrane ASP protein derived from HIV-1 antisense transcription in T cells
}

\author{
Isabelle Clerc ${ }^{1,2,3 \dagger}$, Sylvain Laverdure ${ }^{1,2,3 \dagger}$, Cynthia Torresilla ${ }^{4 \dagger}$, Sébastien Landry ${ }^{4,5}$, Sophie Borel ${ }^{1,2,3}$, \\ Amandine Vargas ${ }^{4}$, Charlotte Arpin-André ${ }^{1,2,3}$, Bernard Gay ${ }^{1,2,3}$, Laurence Briant ${ }^{1,2,3}$, Antoine Gross ${ }^{1,2,3}$, \\ Benoit Barbeau ${ }^{4^{*}}$ and Jean-Michel Mesnard ${ }^{1,2,3^{*}}$
}

\begin{abstract}
Background: Retroviral gene expression generally depends on a full-length transcript that initiates in the $5^{\prime}$ LTR, which is either left unspliced or alternatively spliced. We and others have demonstrated the existence of antisense transcription initiating in the 3' LTR in human lymphotropic retroviruses, including HTLV-1, HTLV-2, and HIV-1. Such transcripts have been postulated to encode antisense proteins important for the establishment of viral infections. The antisense strand of the HIV-1 proviral DNA contains an ORF termed asp, coding for a highly hydrophobic protein. However, although anti-ASP antibodies have been described to be present in HIV-1-infected patients, its in vivo expression requires further support. The objective of this present study was to clearly demonstrate that ASP is effectively expressed in infected T cells and to provide a better characterization of its subcellular localization.

Results: We first investigated the subcellular localization of ASP by transfecting Jurkat T cells with vectors expressing ASP tagged with the Flag epitope to its $\mathrm{N}$-terminus. Using immunofluorescence microscopy, we found that ASP localized to the plasma membrane in transfected Jurkat T cells, but with different staining patterns. In addition to an entire distribution to the plasma membrane, ASP showed an asymmetric localization and could also be detected in membrane connections between two cells. We then infected Jurkat T cells with NL4.3 virus coding for ASP tagged with the Flag epitope at its C-terminal end. By this approach, we were capable of showing that ASP is effectively expressed from the HIV-1 $3^{\prime}$ LTR in infected T cells, with an asymmetric localization of the viral protein at the plasma membrane.
\end{abstract}

Conclusion: These results demonstrate for the first time that ASP can be detected when expressed from fulllength HIV-1 proviral DNA and that its localization is consistent with Jurkat T cells overexpressing ASP.

\section{Background}

Human lymphotropic retroviruses, such as human T-cell leukemia virus type 1 (HTLV-1) or human immunodeficiency virus type 1 (HIV-1), have evolved multiple strategies to direct the synthesis of a complex proteome from a small genome, which involves alternative splicing, internal ribosomal entry sites, ribosomal frameshifting, and leaky scanning [1]. Retroviral genomes are transcribed through a proviral DNA intermediate integrated into the

\footnotetext{
*Correspondence: barbeau.benoit@uqam.ca; jean-michel.mesnard@cpbs.cnrs. fr

+ Contributed equally

'Université Montpellier 1, Centre d'études d'agents Pathogènes et

Biotechnologies pour la Santé (CPBS), Montpellier, France

${ }^{4}$ Université du Québec à Montréal, Département des sciences biologiques

and Centre de recherche BioMed, Montréal, Canada

Full list of author information is available at the end of the article
}

cell chromosome and expressed by the host transcription machinery. All retroviral genes have been thought to be transcribed through a single promoter located in the $5^{\prime}$ long terminal repeat (LTR) of the provirus. However, early studies have described the presence of conserved open reading frames (ORF) in the complementary strand of the HIV-1 and HTLV-1 proviruses, suggesting the existence of viral mRNAs of negative polarity produced from the 3' LTR $[2,3]$. More recently, we and others have conclusively demonstrated the presence of such antisense RNAs in cells infected with HIV-1 or HTLV-1 [4-7].

In the case of HTLV-1, the antisense strand-encoded protein that we have termed HBZ for HTLV-1 bZIP factor $[8]$ is a c-Fos-like nuclear factor $[9,10]$ that attenuates the activation of AP-1 [11-14] and down-regulates viral transcription $[15,16]$. In vivo studies using a rabbit model have

\section{C) Biomed Central}


shown that HBZ is involved in the establishment of chronic viral infections [17], indicating that HBZ could play a key role in the escape of HTLV-1 from the immune system by controlling viral expression $[18,19]$. Interestingly, we have recently demonstrated that HTLV-2 encodes an antisense protein (called APH-2 for antisense protein of HTLV-2) that also represses viral transcription [20].

Although all functional HIV-1 genes are thought to be transcribed from the sense proviral DNA strand only, a very recent study has shown that cryptic epitopes derived from an HIV-1 antisense ORF are generated in infected
CD4+ T lymphocytes [21], confirming the production of viral proteins from antisense transcription. Among the different negative sense ORFs found in HIV-1 [2,6], the asp (for antisense protein [22]) ORF, encoded by the complementary strand to the gp120/gp41 junction of the env gene (Figure 1A), is the most conserved and the longest. Moreover, its presumed ATG initiation codon is also very well preserved. In addition, its position from the 3' LTR is extremely similar to the $h b z$ ORF in HTLV- 1 and the aph-2 ORF in HTLV-2. Asp codes for a highly hydrophobic protein [2] (Figure 1A) that has been found associated with virions released from infected cells [22].

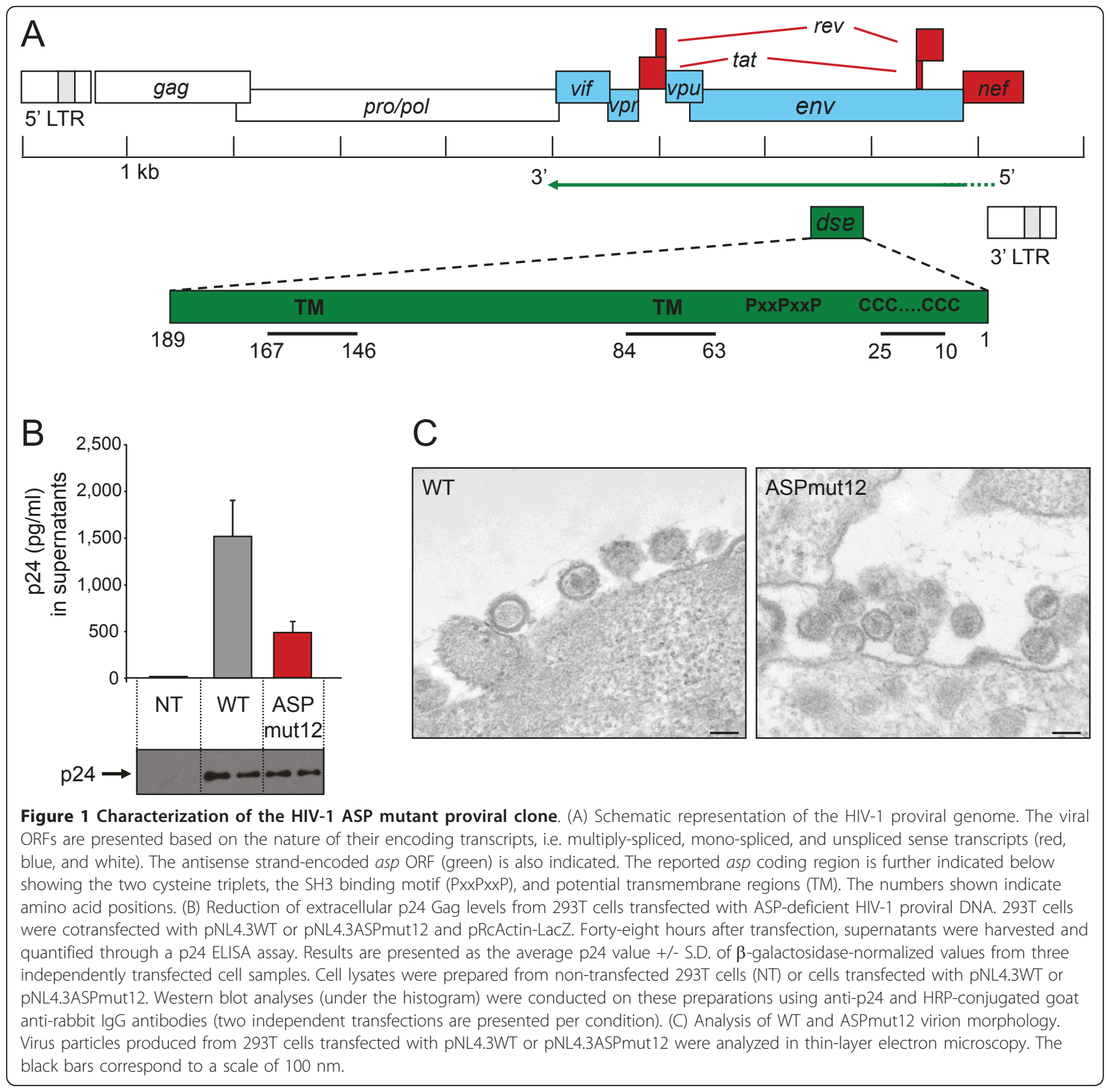


Moreover, the ASP protein has been described to be recognized by antibodies present in patients infected by HIV-1 [23]. Here, we demonstrate for the first time that ASP is expressed in Jurkat T cells infected with a proviral clone, with an asymmetric localization of the viral protein at the plasma membrane.

\section{Results}

\section{Construction and characterization of an HIV-1 ASP mutant proviral clone}

In order to study ASP, we first generated a mutated proviral clone in which a stop codon was inserted in frame to the asp ORF. This mutation resulted in termination of ASP at amino acid 12 of the published sequence [2] without altering the amino acid composition of the Env protein encoded on the sense strand. This resulting pNL4.3ASPmut12 construct was next transfected in $293 \mathrm{~T}$ cells and compared for $\mathrm{p} 24$ production to $293 \mathrm{~T}$ cells transfected with wild type (WT) pNL4.3. Transfected cells showing comparable transfection efficiency were then selected to evaluate their levels of extracellular viral capsid proteins. Interestingly, 293T cells transfected with the mutated proviral DNA showed lower extracellular p24 levels when compared to results obtained with the parental wild-type proviral DNA (Figure 1B). To determine whether p24 expression was also reduced intracellularly, cell lysates from transfected cells were analysed by Western blotting. As shown in Figure 1B, intracellular p24 levels were not affected by the ASP mutation. These data were confirmed in three different experiments, and analyses of p24 signals by densitometry further demonstrated equivalent p 24 levels in cells transfected with the two tested NL4.3 proviral DNA (see the additional file 1, figure S1). Western blot analyses were also performed on the same preparation by using human anti-HIV-1 serum and confirmed that intracellular levels of viral proteins were not affected (data not shown). The effect of the mutation was also investigated on the structure of the viral particle by electron microscopy analysis, and normal-sized mature virions were found in preparations of WT and ASPmut12 particles (Figure 1C). The presence of unambiguous coneshaped nucleoids was also observed in WT and ASPmut12 viruses. In addition, when Jurkat and Sup-T1 cells were infected with WT or ASPmut12 viruses, no significant differences in the levels of extracellular p24 were detected at different times post-infection between both viruses (Figure 2).

The HIV-1 ASP protein localizes to the plasma membrane To better characterize the ASP protein, its expression and localization were analyzed in Jurkat cells. Analysis of its amino acid sequence reveals a highly hydrophobic protein. Hydropathy and immunogenicity plots demonstrate a minimal number of soluble regions and suggest two transmembrane domains extending from amino acid 63 to 84 and amino acid 146-167 (Figure 1A). In its N-terminal region, the ASP sequence also revealed the presence of two conserved cysteine triplets (with a potential palmitoylation site for the first one) and two SH3-binding motifs with a typical proline rich sequence with a PxxP minimal core (Figure 1A). We thus presumed that the presence of potential transmembrane domains could lead to membrane localization of the protein.

To test this hypothesis, Jurkat T cells were transfected with an ASP expression vector in which ASP was tagged with the Flag epitope at its $\mathrm{N}$-terminal end. The transfected cells were co-stained with both FITC-Co-Tx (which binds to GM1 ganglioside, a component of the cell plasma membrane) and anti-Flag antibody and subsequently analysed on a confocal microscope (Figure 3). Image merging of both fluorescent signals confirmed the localization of ASP to the plasma membrane. The

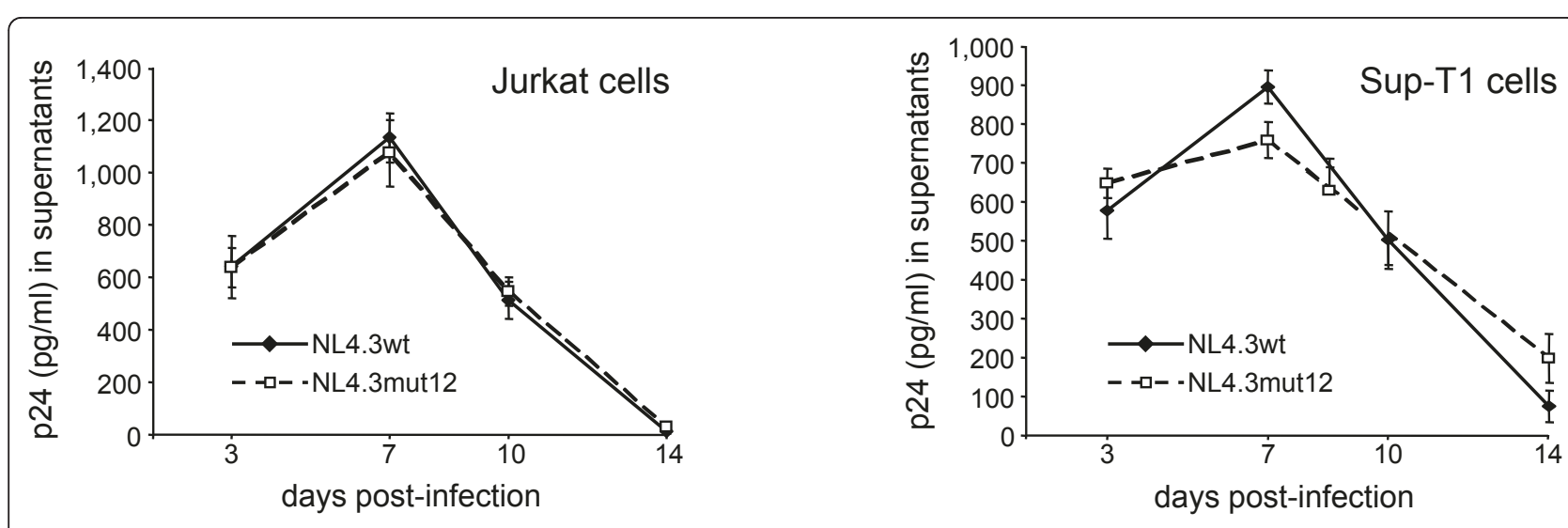

Figure 2 HIV-1 infection of T cells is not affected by the absence of ASP. HIV-1 viral particles harvested from 293T cells transfected with pNL4.3WT or pNL4.3ASPmut12 were used to infect Jurkat (A) and Sup-T1 (B) cells. Extracellular p24 levels were quantified on supernatant from triplicate infected samples and are presented as the mean value +/- S.D. 


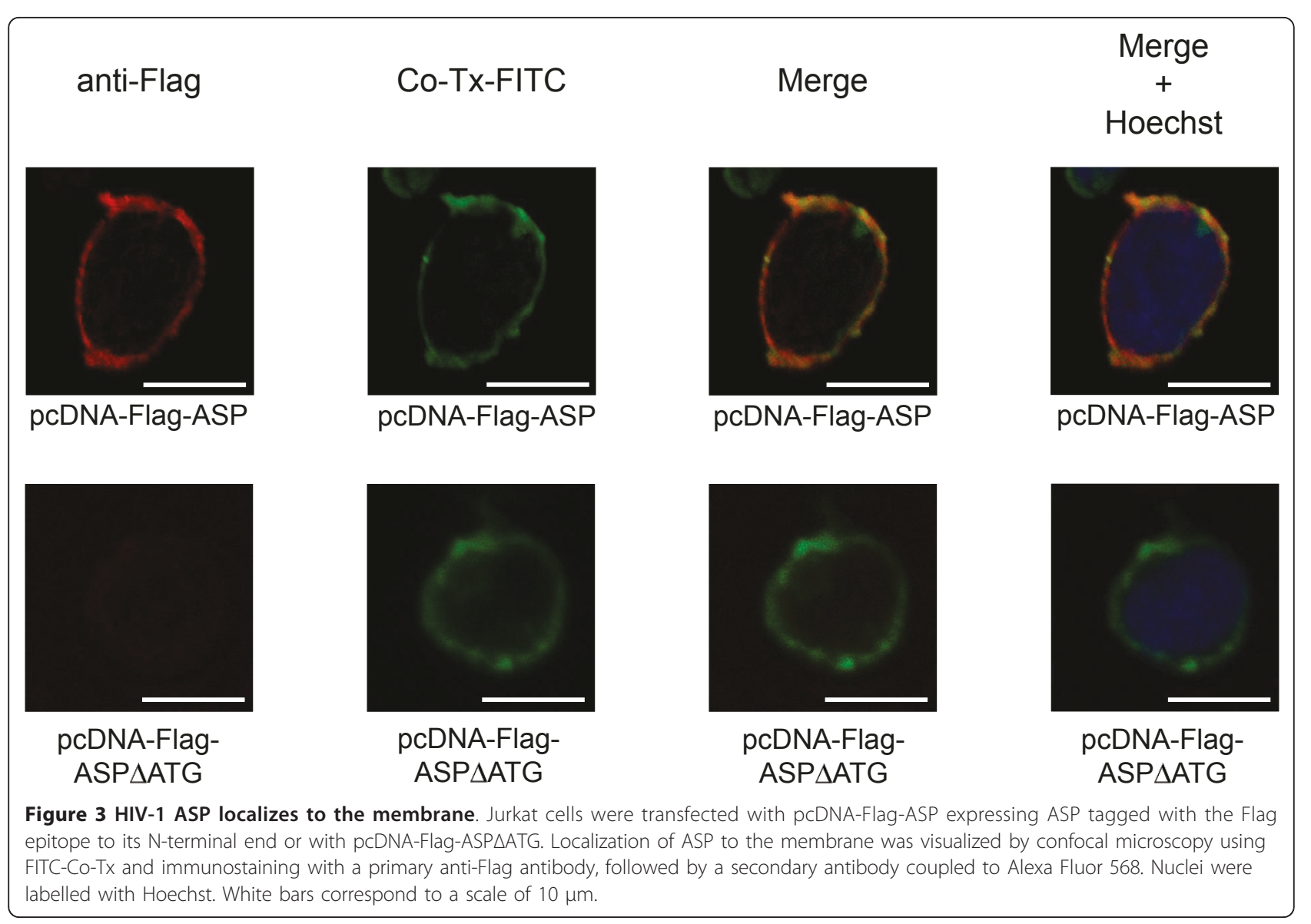

same approach was performed with cells transfected with the pcDNA-Flag-ASP $\triangle \mathrm{ATG}$ expression vector, in which the initiation codon of Flag-ASP was replaced by a stop codon. No specific fluorescent signal was detected with the anti-Flag antibody as illustrated in Figure 3.

ASP localizes differently at the membrane of Jurkat cells Although our results showed that ASP localized to the plasma membrane, two distinct sites were observed. In addition to its unpolarized localization to the plasma membrane (Figure 4A), ASP also showed an asymmetric distribution in ASP-expressing Jurkat cells (Figure 4B). Polarization of ASP to the plasma membrane was found in $44 \% \pm 5$ of transfected cells while unpolarized distribution corresponded to $44 \% \pm 3$ (Figure $4 \mathrm{E}$ ). Moreover, ASP occasionally presented a strong localization into membrane protrusion (Figure $4 \mathrm{C}$ ) corresponding to $12 \% \pm 2$ of transfected cells (Figure 4E). Such staining patterns were not observed in Jurkat cells tranfected with the negative control pcDNA-Flag-ASP $\triangle$ ATG (data not shown). We also analyzed the subcellular localization of an ASP mutant, called ASPmut66, corresponding to the first 65 amino acid residues of ASP, which were thus devoid of both potential transmembrane domains. Compared to the wild type, this mutant showed a different staining profile since ASPmut66 was not localized to the plasma membrane (Figure 4D).

Using this approach, we also detected ASP in membrane connections between two cells as shown in Figure 5A. Based on these results, we further analyzed the distribution of ASP in transfected Jurkat cells seeded on polylysine-covered glass slides at high density, thus favouring cell-to-cell interactions. Interestingly, an intense staining of ASP was found in membrane projections (Figure 5B). Moreover, the ASP staining can highlight a thin and long connection between neighbouring cells (Figure 5C). When similar analyses were performed in Jurkat cells transfected with pEGFP, the fluorescent signal demonstrated a diffuse pattern present not only in intercellular connections, but also in all cellular compartments (Figure 5D). The Scrib (Scribble) protein is a cell membrane-associated protein involved in the regulation of the asymmetric distribution of proteins in T cells $[24,25]$. We then compared the localization of ASP with hScrib in transfected Jurkat cells seeded on polylysine-covered glass slides. As shown in the additional file 2, figure S2, ASP co-localized with endogenous hScrib in membrane projections. 


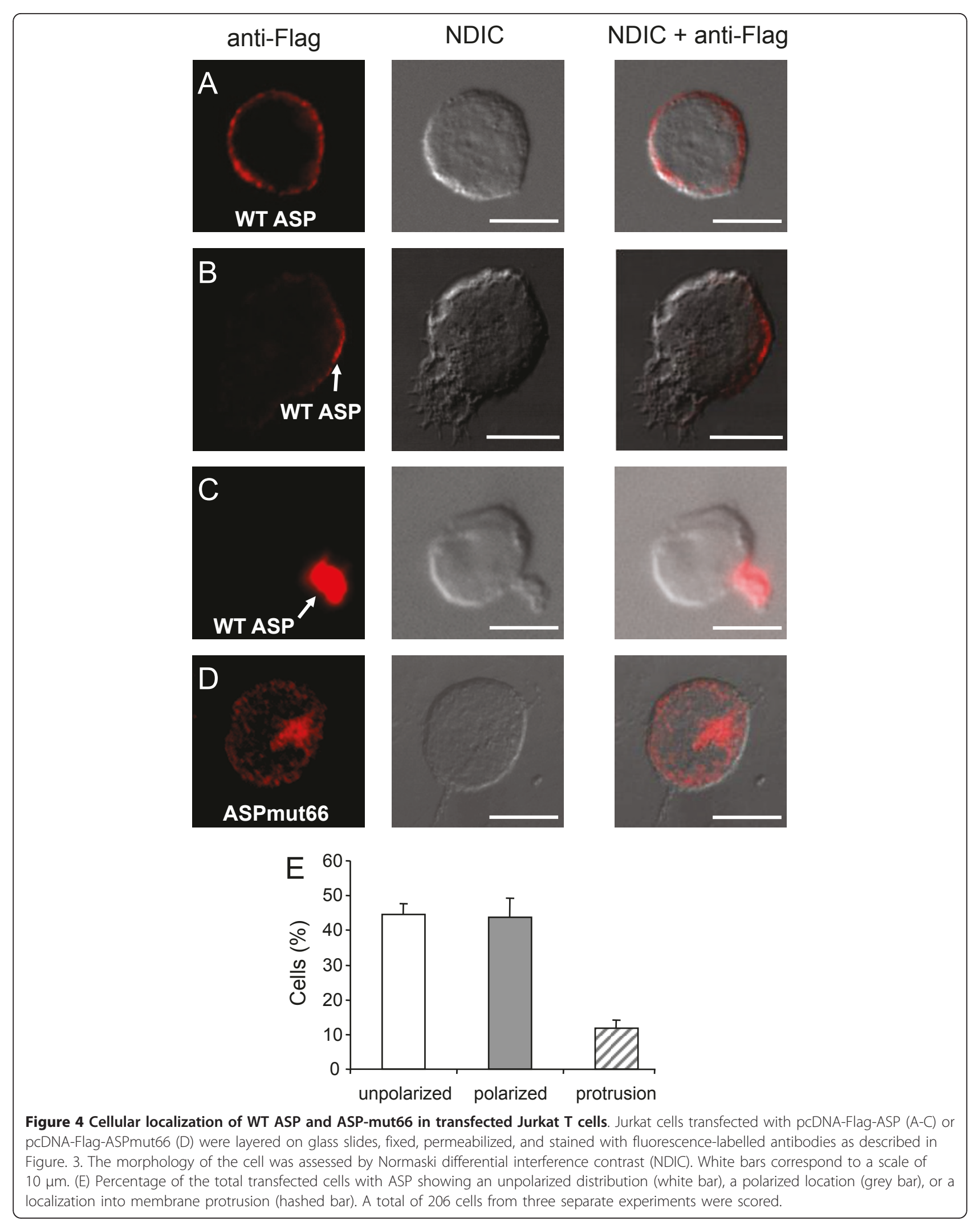




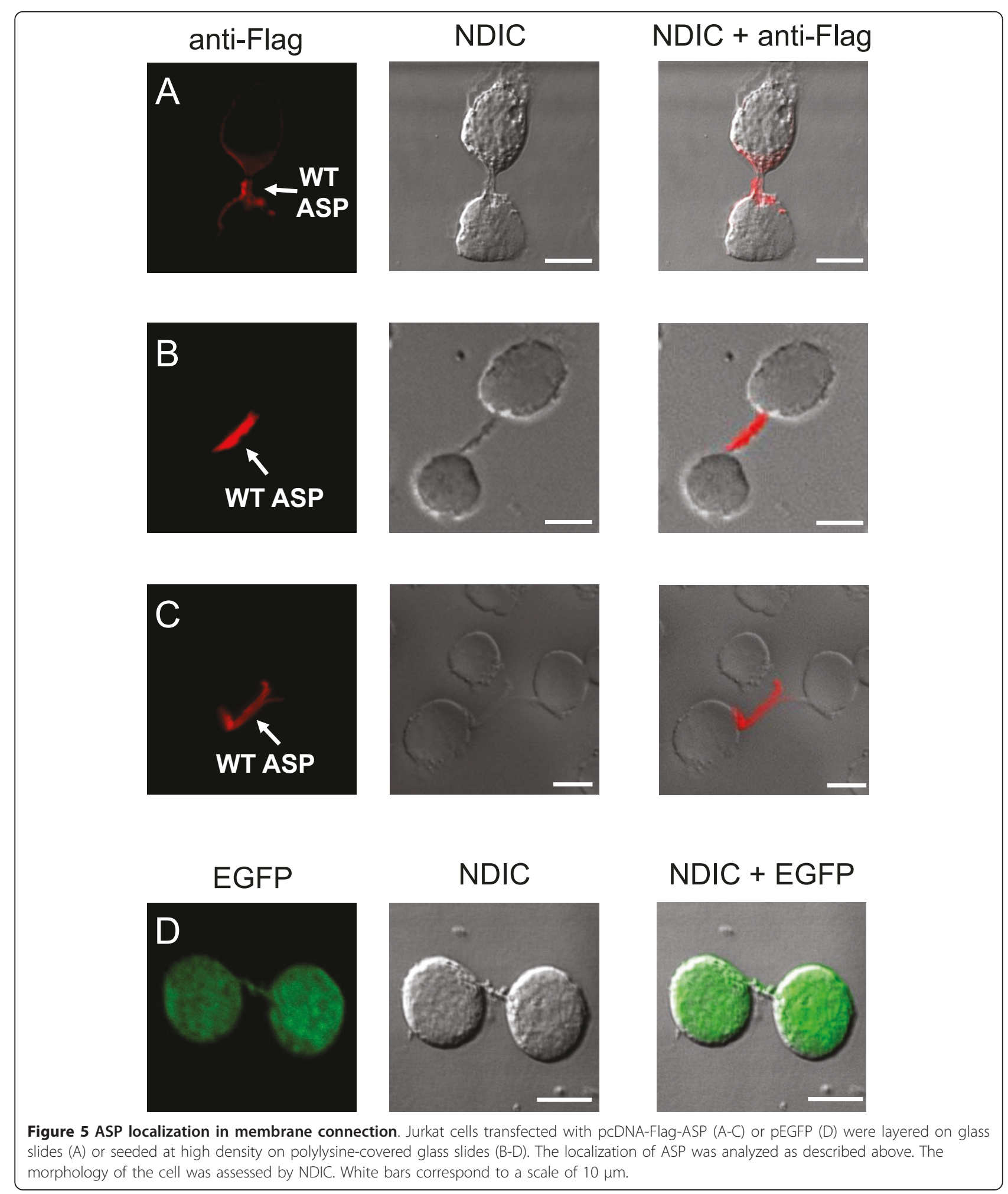

\section{ASP is expressed in infected Jurkat T cells}

Before analyzing the expression of ASP in infected cells, we first compared 5'-LTR-driven sense transcription with the 3'-LTR antisense transcriptional activity in infected Jurkat cells up to 48 hours post infection (hpi). For this experiment, we made use of previously described proviral DNA constructs containing the luciferase reporter gene inserted in the nef coding sequence, 
either in the sense (pNL4.3LucE $\mathrm{R}^{-}$) or antisense direction (pNL4.3AsLucE $\mathrm{R}^{-}$) $[7,26]$. Both molecular proviral clones were separately cotransfected with a VSVg expression vector in $293 \mathrm{~T}$ cells to produce virions pseudotyped with the VSV envelope. Jurkat cells were subsequently infected with an identical infectious viral titer for both types of virions $(\mathrm{MOI}=2)$. As depicted in Figure 6, at $48 \mathrm{hpi}$, luciferase activity was notably lower in Jurkat cells infected with NL4.3AsLucE ${ }^{-} \mathrm{R}^{-}$virions when compared to cells infected with NL4.3LucE $\mathrm{R}^{-}$virions. Nonetheless, a continuous increase in luciferase activity was observed for both viruses and the 3'-LTR antisense activity was the highest at $48 \mathrm{hpi}$.

Next, to detect ASP in infected Jurkat cells, we generated the proviral clone, pNL4.3ASP-Flag, in which ASP was tagged with the Flag epitope at its $\mathrm{C}$-terminal end (Figure 7A); the presence of this tag resulted in termination of Env at amino acid 408. Therefore this molecular proviral clone was cotransfected with a VSVg expression vector in 293T cells to produce pseudotyped virions able to infect Jurkat cells. As determined by our analysis on the 3'-LTR transcriptional activity, expression and localization of ASP were analyzed by fluorescence microscopy at the optimal time, i.e. $48 \mathrm{hpi}$. Although ASP was detected in very few cells, its polarized localization was again confirmed in infected cells (Figure 7B). As negative control, we generated the mutant proviral DNA clone, pNL4.3ASPmut12-Flag, in which the expression of ASPFlag was inhibited by introducing a stop codon at amino acid 12 of ASP as described above (see Figure 1). No staining was detected in Jurkat cells infected with viruses derived from this mutated construct, although Gag- positive cells were observed as frequently as the other tested proviral DNA (Figure 7C and the additional file 3, figure S3).

Taken together, our results demonstrate for the first time that ASP is detected when expressed from fulllength proviral DNA and that its localization is consistent with Jurkat cells overexpressing ASP.

\section{Discussion}

The existence of bidirectional transcription from retrovirus LTRs has been initially suggested based on the identification of conserved ORFs in the antisense strand of their genome, and its demonstration has been mostly focused on human lymphotropic retroviruses. An initial study by Miller [2] had addressed this possibility in HIV1 , and similar ORFs had subsequently been identified on the antisense strand of other retroviruses like HTLV-1 and feline immunodeficiency virus [3,27]. However, the existence of antisense transcription in retroviruses was controversial until the characterization of HBZ in 2002 [8]. Since then, antisense transcription has also been confirmed in HTLV-2 [20] and in gammaretroviruses such as murine leukemia virus [28]. Over the years, transcription initiation has been demonstrated to be a complex process and, in fact, most promoter regions associated to active mammalian genes can transcribe in both sense and antisense directions $[29,30]$. It seems that retroviruses have developed a mechanism to hijack the bidirectional transcription machinery to produce proteins from sense and antisense transcription. The presence of coding genes can probably stimulate elongation by RNA polymerase II either in the sense direction from the 5' LTR,

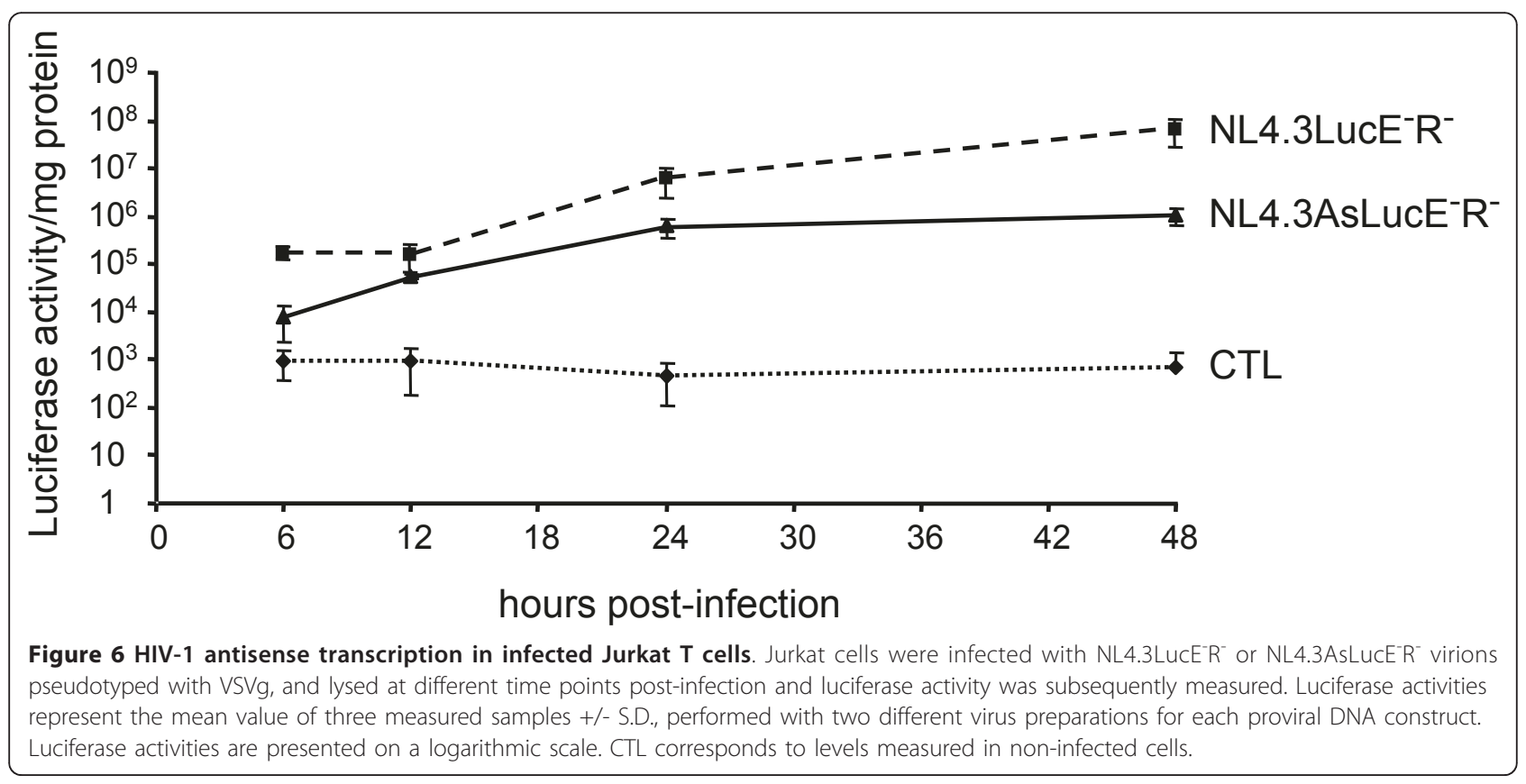




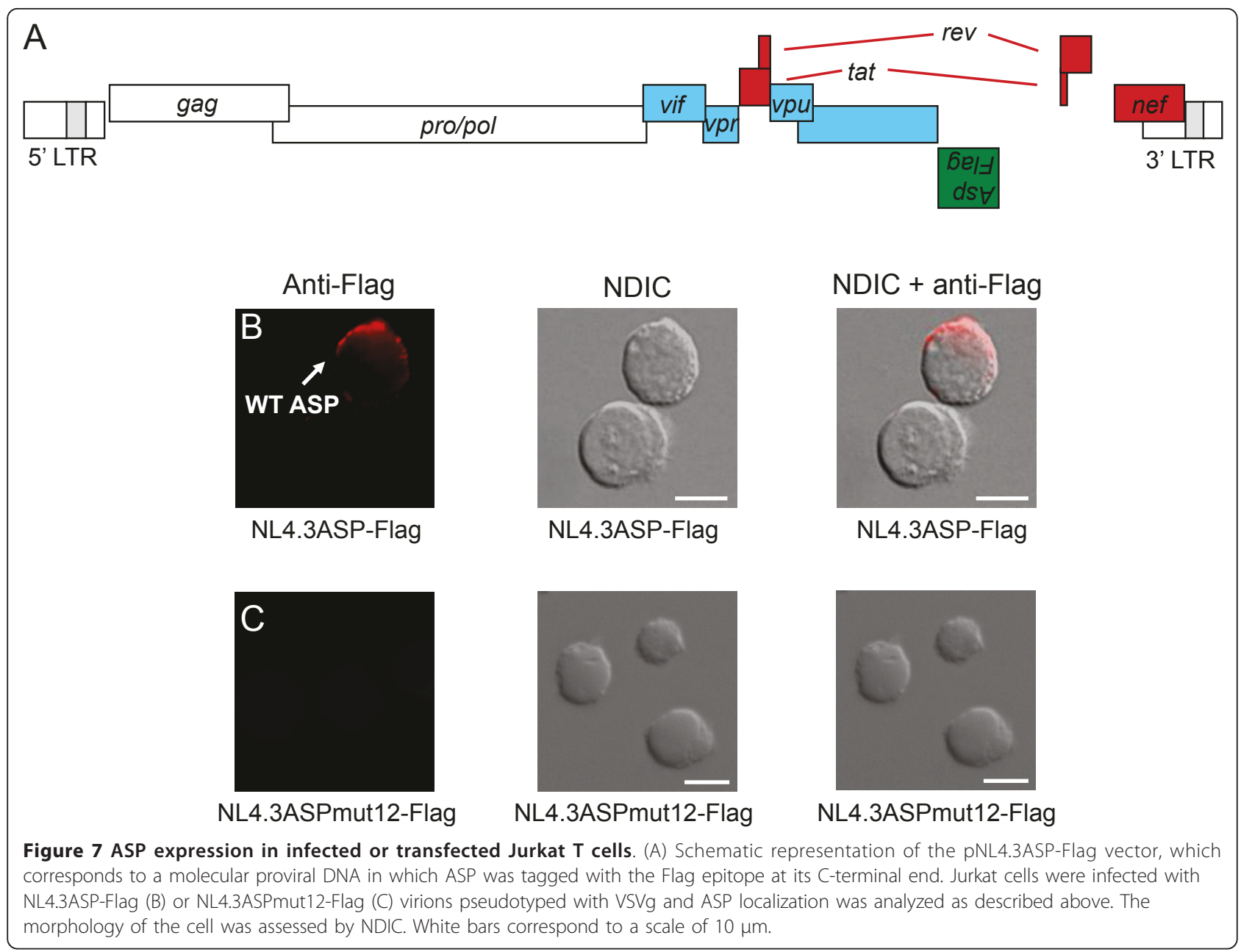

or in the antisense direction from the 3' LTR. Such a mechanism allows the synthesis of a complex proteome from the proviral genome integrated into the cell chromosome.

Although the synthesis of proteins from antisense transcripts has been clearly demonstrated in the case of HTLV-1 and $-2[8,20]$, this possibility remains debated for HIV-1. Antisense RNA was however identified in various cell lines chronically infected with HIV-1 [7,23,31]. By RACE analyses, we have recently identified several transcription initiation sites near the 5' border of the 3' LTR and a polyA signal located at $2.4 \mathrm{~kb}$ distance from the ASP stop codon [7]. Such transcripts are potentially templates encoding the ASP protein. Indeed, it has been found that translation of the in vitro-synthesized antisense RNA yielded a protein with an apparent molecular weight of $19 \mathrm{kDa}$ in SDS-PAGE [23] corresponding to the theoretical molecular weight of ASP. Moreover, this report further described the presence of antibodies against ASP in several sera of HIV-1-infected patients [23]. Interestingly, very recent results support the notion that epitopes derived from antisense transcripts serve as CD8 T-cell targets in HIV-1 infection [21]. Taken together, all these data suggest that the HIV-1 ASP protein should be expressed in vivo. However, its detection through Western blot analysis from cellular extracts has not yet been possible. Different reasons can explain the lack of detection of the ASP protein. First of all, antisense retroviral proteins are poorly expressed in vivo $[8,20]$. Levels of antisense transcripts can be 30 to 1000 folds lower than that of sense transcripts [7]. In addition, the negative effect of certain sequences on RNA stability is well known in the case of HIV-1 sense transcripts. For instance, $\mathrm{Vpu}$ and Vif proteins are poorly expressed from expression vectors and generation of codon-optimized viral cDNAs can overcome this limitation [32]. Indeed, ASP expression can be improved by codon optimization of its coding sequence (B.B., personal communication). A second concern for the detection of ASP is related to its structure. In this paper, we demonstrate that ASP is localized to the plasma membrane. This localization is consistent with the predicted structure of ASP, which is a 
highly hydrophobic protein displaying two potential $\alpha$ helical transmembrane segments. Furthermore, the ASPmut66 protein (deleted of both potential transmembrane domains) did not localize to the plasma membrane, confirming the predicted structure. Its inherent membrane-bound nature makes the characterization of this protein particularly difficult and likely requires special experimental conditions [33]. In addition, characterization of membrane proteins is very difficult because they are usually poorly abundant. All these issues concerning membrane proteins explain the great disparity between current knowledge of soluble versus membrane proteins.

In this paper, by using a strategy different from Western blot analyses, we clearly demonstrate for the first time that ASP is expressed in infected Jurkat T cells. By using fluorescence microscopy, we have first characterized the distribution of ASP tagged with the Flag epitope in Jurkat cells. We then compared ASP localization in these conditions with that in Jurkat cells infected with a proviral clone in which ASP was tagged with the Flag epitope to its $\mathrm{C}$-terminus. We could indeed confirm the polarized localization profile of ASP in infected cells. As expected, this staining pattern was abolished by introducing a stop codon in the asp ORF. By using immunoelectron microscopy, expression of ASP has been previously analyzed in HIV-1-infected Sup-T1 cells and the viral protein appeared to concentrate in the nucleus and in the cytoplasm [22]. ASP was also detected in the activated $\mathrm{ACH}-2$ cell line, a chronically infected $\mathrm{T}$ cell line. In this study, ASP localized in several cell compartments including the nucleus, the nucleolus, and the mitochondria but not at the plasma membrane [22]. The presence of ASP was detected in the cytoplasm and the nucleus in the vicinity of the cell membranes. However, the nucleolar localization of ASP is unexpected since the nucleolus is a non-membrane bound structure. In our infection experiments, we have never detected ASP associated to the nucleus or the nucleolus. We are unable to explain this discrepancy between the two approaches.

At the moment, the function of ASP remains mysterious. In our studies, we have noted a significant reduction in the level of extracellular p24 production from 293T cells transfected with the mutant pNL4.3ASPmut12 compared to the parental wild-type proviral DNA, but we have been unable to reproduce these results in Jurkat and SupT1 cells infected with virions produced with the same mutant. This difference could be explained by the method used to introduce the viral genome into cells. In the case of infection, integration of retroviral DNA into the host genome is an obligatory step for viral protein expression. Depending on the chromosomal location of the integrated provirus, LTR-mediated transcription may vary from 0 - to 70-fold. At the moment, we do not know whether ASP is more expressed in transfected 293T cells than in infected
Jurkat cells. Similarly, regulation of ASP expression during viral life cycle remains unclear. In the case of HTLV-1, kinetic analysis revealed that antisense transcription was expressed at a low level early after infection and continued to increase before reaching a plateau, showing an inverse correlation between sense/antisense transcription over time [34]. We do not observe a similar trend in the case of HIV-1 but experiments are currently in progress to study the regulation of antisense transcription in primary cells. It is thereby difficult to draw conclusions concerning the function of ASP.

\section{Conclusion}

We demonstrate for the first time that ASP can be produced in infected Jurkat $\mathrm{T}$ cells. The in vivo detection of ASP gives a novel tool to better understand how HIV-1 is involved in the development of immunodeficiency.

\section{Methods}

\section{Plasmids and antibodies}

The pNL4.3 HIV-1 proviral DNA was obtained from the NIH AIDS Research and Reference Reagent Program (Germantown MD). To produce the pNL4.3-ASPmut12 construct, a NdeI/BamHI fragment containing the asp sequence was first cloned in a similarly digested pGL3 basic vector. Using primers 24-8 (5'-GTTGCAACTCACAGTCTGGGGCAT-3') and 24-7 (5'-AGATGCTGTTGAGCCTC AATAGCC-3'; the mutated nucleotide is indicated in bold), reverse PCR was used to mutate the cysteine residue in position 12 into a stop codon (TGC into TGA). Sequencing of the entire NdeI/BamHI fragment confirmed the specific mutation after which the fragment was cloned back in the pNL4.3 DNA to replace the wild type segment. The pNL4.3LucE ${ }^{-} \mathrm{R}^{-}$vector (containing the luciferase reporter gene and deficient for Env and Vpr synthesis [26]) was generously provided by Dr N.R. Landau. The pNL4.3AsLucE $\mathrm{R}^{-}$vector has previously been described [7]. The Flag-ASP, Flag-ASP $\triangle \mathrm{ATG}$, and FlagASPmut66 cDNA fragments were generated by PCR amplification using Deep Vent DNA polymerase and specific sense and antisense primers. The nucleotide sequence coding for the Flag epitope (DYKDDDDK) has been inserted in the sequence of the sense primer. The synthesized cDNA was inserted into the BamHI/EcoRI cloning sites of the linearized pcDNA3ZEO vector. To generate the pNL4.3ASP-Flag construct, the NL4.3-derived NdeI/ BamHI fragment cloned in pGL3 basic was used to add NcoI and $\mathrm{XbaI}$ sites and displace the stop codon at the 3' end of the ASP ORF by reverse PCR with the following primers: 5'-GCTCTAGATAGAAAAATTCCCCTCCACAATTAAAACTG-3' (sense) and 5'-GTCCATGGCTGTAATTCAACACAACTGTTTAATAGTAC-3' (antisense). Primers permitting the addition of a Flag tag at the $\mathrm{COOH}$ end of the ASP ORF, 5'-CATGGGACTACA 
AGGACGACGACGACAGT-3' (sense) and 5'-CTAGACTTGTCGTCGTCGTCCTTGTAGTCC-3' (antisense), were annealed and inserted in frame at the 3' end of the ASP ORF after NcoI/Xba I digestion. The resulting Flag-tagged ASP ORF was reinserted in the NL4.3 proviral DNA using the NdeI/BamHI sites. The 5'LTR-deleted pNL4.3ASP-Flag $\Delta 5^{\prime}$ LTR construct was then generated by NarI digestion and self ligation. pRcActin-lacZ contains the $\beta$-galactosidase gene under the control of the human $\beta$-actin promoter.

Mouse anti-p24 antibody was purchased from Abcam, while goat anti-mouse IgG was bought from GE Healthcare. Mouse anti-Gag KC57-RD1 antibody was purchased from Beckman-Coulter. We have obtained the mouse anti-Flag antibody from Sigma and the rabbit anti-hScrib (H-300) antibody from Santa Cruz Biotechnology, Inc.

\section{Transfection, infection, and detection of viral p24 capsid antigen}

Transfection experiments in 293T cells were conducted as previously described [35]. Briefly, 293T cells $\left(3 \times 10^{5}\right)$ were plated $24 \mathrm{~h}$ prior to transfection in a 6 -well plate and were next transfected through the calcium phosphate protocol with $3 \mu \mathrm{g}$ wild-type or ASP-deficient pNL4.3 proviral DNA construct along with $0.5 \mu \mathrm{g}$ pRcActin-lacZ (used to normalize transfection experiments). At $48 \mathrm{~h}$ post-transfection, supernatants were harvested and levels of the HIV-1 p24 capsid protein was evaluated by a p24specific ELISA assay as previously described [36]. All experiments were performed in triplicates and final p24 levels are represented as the average of independent triplicate transfection experiments after $\beta$-galactosidase normalization. Jurkat and Sup-T1 cells $\left(1 \times 10^{6}\right.$ cells $)$ were infected with harvested wild-type and ASP-deficient NL4.3 viruses (100 ng p24) in RPMI supplemented with $10 \%$ FCS. Extracellular p24 levels were quantified by ELISA at days 3, 7, 10 and 14, and represent the mean values +/- S.D of three independently infected samples.

\section{Western blot}

Transfected 293T cells were washed in PBS and proteins

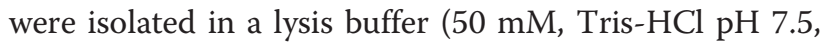
$150 \mathrm{mM} \mathrm{Nacl}, 0.5 \% \mathrm{NP}-40$, and protease inhibitor cocktail in tablets). Protein concentrations were then quantified with the BCA protein assay (Thermo Fisher Scientic Inc.). Cellular extracts were migrated on an 8.5\% SDSPAGE and transferred on a PVDF membrane. Membranes were next blocked in 3\% BSA and incubated with a polyclonal anti-p24 antibody $(1 / 1000)$ and were further incubated with horseradish peroxidase-conjugated goat anti-rabbit antibody (1/1000). Signals were detected with the BM chemiluminescence blotting substrate kit (Roche Diagnostics) and membranes were subsequently exposed on an ECL high performance chemiluminescence film (Amersham Biosciences). Densitometric analyses were conducted on three independent Western blot analyses from these transfected cells and mean values +/- S.D. were determined.

\section{Electron microscopy analysis}

Cells were fixed in situ with $2.5 \%$ glutaraldehyde in 100 $\mathrm{mM}$ cacodylate buffer $\mathrm{pH} 7.4$ for $3 \mathrm{~h}$ at $4^{\circ} \mathrm{C}$. After three washes in cacodylate buffer, cells were postfixed with $2 \%$ osmium tetroxide for $1 \mathrm{~h}$ at room temperature and washed in cacodylate buffer. A third fixation in $0.5 \%$ tannic acid was performed at room temperature. After extensive washes in $0.1 \mathrm{M}$ Sorensen phosphate buffer $\mathrm{pH}$ 7.2, cells were included in a fibrin clot. Cells were then dehydrated and embedded in epony resin (Embed812; Electron Microscopy Sciences). Sections were counterstained with uranyl acetate and lead citrate alkaline and examined with the transmission electron microscope Hitachi H7100 TEM.

\section{Fluorescence microscopy analysis}

Jurkat cells were cultured in RPMI supplemented with $10 \%$ FCS. For transient-transfection assays, cells $\left(10^{6}\right)$ were transfected with $2 \mu \mathrm{g}$ plasmids with the device Nucleofector II according to the manufacturer's instructions (Amaxa Biosystems, Lonza). After 48 h, cells were pelleted, washed twice in PBS and overlaid for $30 \mathrm{~min}$ at $37^{\circ} \mathrm{C}$ on glass slides coated or not with polylysine. Cells were then fixed with $4 \%$ formalin for $10 \mathrm{~min}$, followed by a treatment of $5 \mathrm{~min}$ with $\mathrm{NH} 4 \mathrm{Cl} 5 \mathrm{mM}$ to remove excess of formaldehyde. Cells were next permeabilized with $0.1 \%$ Triton for $7 \mathrm{~min}$. Fixed cells were subsequently incubated with a blocking solution (PBS containing 5\% FCS) and then with the primary antibody (mouse anti-Flag M2 antibody; Sigma) for $1.5 \mathrm{~h}$ at $37^{\circ} \mathrm{C}$. After several washes with PBS, the cells were incubated with the secondary antibody coupled to Alexa Fluor 568 (Invitrogen) for $45 \mathrm{~min}$ at room temperature. If necessary the nuclei were stained with Hoechst (Sigma). In certain experiments, cells were stained with fluorescein isothiocyanate-conjugated cholera toxin B (FITC-Co-Tx; Sigma) by incubating the cells with $5 \mu \mathrm{g} / \mathrm{ml}$ of FITC$\mathrm{Co}-\mathrm{Tx}$ overnight at $4^{\circ} \mathrm{C}$ before fixation. Coverslips were mounted with Prolong GOLD (Invitrogen) for direct observation as previously described [25]. Fluorescence images were acquired by a fluorescence microscope (model DC250 Leica), and analysis of the green, red, and blue fluorescence in colocalization experiments was performed with a SP2 Leica confocal microscope.

\section{Infection and luciferase assays}

To produce the pseudotyped HIV-1 particles, 293T cells were plated $16 \mathrm{~h}$ before transfection $\left(7 \times 10^{6}\right.$ cells per 
flask into six $75 \mathrm{~cm}^{2}$ flasks) and then transfected with the different pNL4.3 proviral DNA $(30 \mu \mathrm{g})$ and the Vesicular Stomatitis Virus envelope (VSVg) expression vector $(18 \mu \mathrm{g})$ using the jetPEITM transfection reagent (Qbiogene) according to manufacturer's instructions. Five hours after transfection, cells were cultured for 48 $\mathrm{h}$ in serum-free DMEM. Viruses were then collected by filtering the culture media through a $0.45 \mu \mathrm{m}$ pore size cellulose acetate membrane and the filtrate was spun down at $100000 \mathrm{~g}$ at $4^{\circ} \mathrm{C}$ during $2 \mathrm{~h}$. After centrifugation, the supernatant was removed and the viral pellet was resuspended in 100-500 $\mu$ l RPMI supplemented with 5\% FCS. Virus stocks were then aliquoted and frozen at $-80^{\circ} \mathrm{C}$ for future use. All virus stocks underwent a single freeze-thaw cycle before use in infection studies. Infectious virus titer was determined by infecting Jurkat cells $\left(10^{6} /\right.$ well in a 24 -well plate) with two-fold serial dilution of virus stocks. At 48 hours post-infection (hpi), the percentage of infected cells was determined by intracellular staining of HIV-1 Gag with KC57-RD1 antibodies followed by analysis with an Epics XL flow cytometer (Beckman-Coulter). Jurkat cells $\left(10^{6} /\right.$ well) were infected with an identical multiplicity of infection $(\mathrm{MOI}=2)$ for virus stocks. Luciferase assays were performed in an automated luminometer (Contro $\mathrm{XS}^{3}$ LB960, Berthold technologies) with the Genofax A kit (Yelen, Ensue la Redonne) according to the manufacturer's instructions.

\section{Additional material}

\section{Additional file 1: Figure S1. Analyses of p24 signals by}

densitometry. Densitometric analyses were used to quantify p24 levels and are expressed as a ratio of p24 over GAPDH. Mean values +/- S.D. were calculated from three independent transfection and Western blot analyses.

Additional file 2: Figure S2. ASP colocalizes with hScrib. Microscopy analysis of endogenous hScrib was performed in Jurkat cells transfected with pcDNA-Flag-ASP already shown in Figure. 5C. Jurkat cells were stained with the rabbit anti-hScrib antibody and goat anti-rabbit immunoglobulin $\mathrm{G}$ antibody coupled to FITC while the localization of ASP was analyzed as already described. For localization, analysis of green (anti-hScrib), red (anti-Flag), and merged fluorescence was performed with a confocal microscope. Nuclei were labelled with Hoechst. White bars correspond to a scale of $10 \mu \mathrm{m}$.

Additional file 3: Figure S3. Gag expression in Jurkat T cells infected with NL4.3ASPmut12-Flag. Jurkat cells were infected (A) or not (B) with NL4.3ASPmut12-Flag and infection was confirmed by intracellular staining of HIV-1 Gag with KC57-RD1 antibodies and analyzed as already described.

\section{Acknowledgements}

We thank Lucile Espert for helpful discussions. Confocal microscopy was performed by the Service de Cytométrie at the Centre Régional d'Imagerie Cellulaire (CRIC) in Montpellier. This work was supported by institutional grants from the Centre National de la Recherche Scientifique (CNRS) and the Université Montpellier 1 (UM 1), a grant to J.M.M. from the CNRS for an international project of scientific cooperation, a grant to J.M.M. from the
Agence Nationale de Recherches sur le Sida et les hépatites virales (ANRS) and a grant to B.B. from The Canadian Foundation for AIDS Research (CanFAR). I.C. was supported by ANRS and Sidaction, S.L. and S.B. are supported by fellowships from the Ministère de I'Enseignement Supérieur et de la Recherche.

\section{Author details}

'Université Montpellier 1, Centre d'études d'agents Pathogènes et Biotechnologies pour la Santé (CPBS), Montpellier, France. ${ }^{2}$ CNRS, UM5236, CPBS, F-34965 Montpellier, France. ${ }^{3}$ Université Montpellier 2, CPBS, F-34095 Montpellier, France. ${ }^{4}$ Université du Québec à Montréal, Département des sciences biologiques and Centre de recherche BioMed, Montréal, Canada.

${ }^{5}$ Salk Institute for Biological Studies, San Diego, USA.

\section{Authors' contributions}

Contribution: $A G, B B, L B$, and JMM. designed the research; $I C, S L, C T, S L, S B$, $A V, C A A$, and BG. performed research and collected data, BB and JMM analyzed data and wrote the paper. All authors read and approved the final manuscript.

\section{Competing interests}

The authors declare that they have no competing interests.

Received: 10 May 2011 Accepted: 19 September 2011

Published: 19 September 2011

\section{References}

1. Bolinger C, Boris-Lawrie K: Mechanisms employed by retroviruses to exploit host factors for translational control of a complicated proteome. Retrovirology 2009, 6:8.

2. Miller RH: Human immunodeficiency virus may encode a novel protein on the genomic DNA plus strand. Science 1988, 239:1420-1422.

3. Larocca D, Chao LA, Seto MH, Brunck TK: Human T-cell leukemia virus minus strand transcription in infected T-cells. Bioch Biophys Res Comm 1989, 163:1006-1013.

4. Cavanagh M-H, Landry S, Audet B, Arpin-Andre C, Hivin P, Paré M-E, Thete J, Wattel E, Marriott SJ, Mesnard J-M, et al: HTLV-I antisense transcripts initiating in the $3^{\prime} L T R$ are alternatively spliced and polyadenylated. Retrovirology 2006, 3:15.

5. Murata K, Hayashibara T, Sugahara K, Uemura A, Yamaguchi T, Harasawa H, Hasegawa H, Tsuruda K, Okazaki T, Koji T, et al: A novel alternative splicing isoform of human T-cell leukemia virus type $1 \mathrm{bZIP}$ factor (HBZ-SI) targets distinct subnuclear localization. J Virol 2006, 80:2495-2505.

6. Ludwig LB, Ambrus JL, Krawczyk KA, Sharma S, Brooks S, Hsiao CB, Schwartz SA: Human Immunodeficiency Virus-Type 1 LTR DNA contains an intrinsic gene producing antisense RNA and protein products. Retrovirology 2006, 3:80.

7. Landry S, Halin M, Lefort S, Audet B, Vaquero C, Mesnard JM, Barbeau B: Detection, characterization and regulation of antisense transcripts in HIV-1. Retrovirology 2007, 4:71.

8. Gaudray G, Gachon F, Basbous J, Biard-Piechaczyk M, Devaux C, Mesnard JM: The complementary strand of HTLV-1 RNA genome encodes a bZIP transcription factor that down-regulates the viral transcription. J Virol 2002, 76:12813-12822.

9. Hivin $P$, Frédéric M, Arpin-André C, Basbous J, Gay B, Thébault $\mathrm{S}$, Mesnard JM: Nuclear localization of HTLV-I bZIP factor (HBZ) is mediated by three distinct motifs. J Cell Science 2005, 118:1355-1362.

10. Hivin P, Arpin-André C, Clerc I, Barbeau B, Mesnard JM: A modified version of a Fos-associated cluster in HBZ affects Jun transcriptional potency. Nucleic Acids Res 2006, 34:2761-2772.

11. Basbous J, Arpin C, Gaudray G, Piechaczyk M, Devaux C, Mesnard JM: HBZ factor of HTLV-I dimerizes with transcription factors JunB and c-Jun and modulates their transcriptional activity. J Biol Chem 2003, 278:43620-43627.

12. Matsumoto J, Ohshima T, Isono O, Shimotohno K: HTLV-1 HBZ suppresses AP- 1 activity by impairing both the DNA-binding ability and the stability of c-Jun protein. Oncogene 2005, 24:1001-1010.

13. Hivin P, Basbous J, Raymond F, Henaff D, Arpin-Andre C, RobertHebmann V, Barbeau B, Mesnard JM: The HBZ-SP1 isoform of human Tcell leukemia virus type I represses JunB activity by sequestration into nuclear bodies. Retrovirology 2007, 4:14. 
14. Clerc I, Hivin P, Rubbo PA, Lemasson I, Barbeau B, Mesnard JM: Propensity for HBZ-SP1 isoform of HTLV-I to inhibit c-Jun activity correlates with sequestration of c-Jun into nuclear bodies rather than inhibition of its DNA-binding activity. Virology 2009, 391:195-202.

15. Lemasson I, Lewis MR, Polakowski N, Hivin P, Cavanagh MH, Thebault S, Barbeau B, Nyborg JK, Mesnard JM: Human T-cell leukemia virus type 1 (HTLV-1) bZIP protein interacts with the cellular transcription factor CREB to inhibit HTLV-1 transcription. J Virol 2007, 81:1543-1553.

16. Clerc I, Polakowski N, Andre-Arpin C, Cook P, Barbeau B, Mesnard J-M, Lemasson I: An interaction between the human T cell leukemia virus type 1 basic leucine zipper factor (HBZ) and the KIX domain of p300/ CBP contributes to the down-regulation of tax-dependent viral transcription by HBZ. J Biol Chem 2008, 283:23903-23913.

17. Arnold J, Yamamoto B, Li M, Phipps AJ, Younis I, Lairmore MD, Green PL: Enhancement of infectivity and persistence in vivo by $\mathrm{HBZ}$, a natural antisense coded protein of HTLV-1. Blood 2006, 107:3976-3982.

18. Mesnard JM, Barbeau B, Devaux C: HBZ, a new important player in the mystery of Adult-T- cell leukemia. Blood 2006, 108:3979-3982.

19. Matsuoka M, Green PL: The HBZ gene, a key player in HTLV-1 pathogenesis. Retrovirology 2009, 6:71.

20. Halin M, Douceron E, Clerc I, Journo C, Ko NL, Landry S, Murphy EL, Gessain A, Lemasson I, Mesnard JM, et al: Human T-cell leukemia virus type 2 produces a spliced antisense transcript encoding a protein that lacks a classical bZIP domain but still inhibits Tax2-mediated transcription. Blood 2009, 114:2427-2437.

21. Bansal A, Carlson J, Yan J, Akinsiku OT, Schaefer M, Sabbaj S, Bet A, Levy DN, Heath S, Tang J, et al: CD8 T cell response and evolutionary pressure to HIV-1 cryptic epitopes derived from antisense transcription. J Exp Med 2010, 207:51-59.

22. Briquet $S$, Vaquero $C$ : Immunolocalization studies of an antisense protein in HIV-1-infected cells and viral particles. Virology 2002, 292:177-184.

23. Vanhee-Brossollet C, Thoreau $H$, Serpente $N$, D'Auriol L, Levy JP, Vaquero C: A natural antisense RNA derived from the HIV-1 env gene encodes a protein which is recognized by circulating antibodies of HIV+ individuals. Virology 1995, 206:196-202.

24. Ludford-Menting MJ, Oliaro J, Sarcibegovic F, Cheah ETY, Pedersen N, Thomas SJ, Pasam A, lazzolino R, Dow LE, Waterhouse NJ, et al: A network of PDZ-containing proteins regulates $\mathrm{T}$ cell polarity and morphology during migration and immunological synapse formation. Immunity 2005, 22:737-748.

25. Arpin-Andre C, Mesnard JM: The PDZ domain-binding motif of the human $\mathrm{T}$ cell leukemia virus type 1 Tax protein induces mislocalization of the tumor suppressor hScrib in T cells. J Biol Chem 2007, 282:33132-33141.

26. Connor $\mathrm{Rl}$, Chen BK, Choe S, Landau NR: Vpr is required for efficient replication of human immunodeficiency virus type-1 in mononuclear phagocytes. Virology 1995, 206:935-944.

27. Briquet S, Richardson J, Vanhee-Brossollet C, Vaquero C: Natural antisense transcripts are detected in different cell lines and tissues of cats infected with feline immunodeficiency virus. Gene 2001, 267:157-164.

28. Rasmussen MH, Ballarin-Gonzalez B, Liu J, Lassen LB, Fuchtbauer A, Fuchtbauer EM, Nielsen AL, Pedersen FS: Antisense transcription in gammaretroviruses as a mechanism of insertional activation of host genes. J Virol 2010, 84:3780-3788.

29. Yang MQ, Elnitski LL: Diversity of core promoter elements comprising human bidirectional promoters. BMC Genomics 2008, 9:S3.

30. Seila AC, Core LJ, Lis JT, Sharp PA: Divergent transcription: a new feature of active promoters. Cell Cycle 2009, 8(1):2557-2564

31. Michael NL, Vahey MT, d'Arcy L, Ehrenberg PK, Mosca JD, Rappaport J, Redfield RR: Negative-strand RNA transcripts are produced in human immunodeficiency virus type 1-infected cells and patients by a nove promoter downregulated by Tat. J Virol 1994, 68:979-987.

32. Nguyen $K L$, llano $M$, Akari $H$, Miyagi E, Poeschla EM, Strebel K, Bour S: Codon optimization of the HIV-1 vpu and vif genes stabilizes their mRNA and allows for highly efficient Rev-independent expression. Virology 2004, 319:163-175.

33. Arinaminpathy $Y$, Khurana E, Engelman DM, Gerstein MB: Computational analysis of membrane proteins: the largest class of drug targets. Drug Discov Today 2009, 14:1130-1135.
34. Li M, Kesic M, Yin H, Yu L, Green PL: Kinetic analysis of human T-cell leukemia virus type 1 gene expression in cell culture and infected animals. J Virol 2009, 83:3788-3797.

35. Vargas A, Moreau J, Landry S, LeBellego F, Toufaily C, Rassart E, Lafond J, Barbeau B: Syncytin-2 plays an important role in the fusion of human trophoblast cells. J Mol Biol 2009, 392:301-318.

36. Fortin JF, Cantin R, Lamontagne G, Tremblay M: Host-derived ICAM-1 glycoproteins incorporated on human immunodeficiency virus type 1 are biologically active and enhance viral infectivity. J Virol 1997, 71:3588-3596.

doi:10.1186/1742-4690-8-74

Cite this article as: Clerc et al:: Polarized expression of the membrane ASP protein derived from HIV-1 antisense transcription in T cells. Retrovirology 2011 8:74.

\section{Submit your next manuscript to BioMed Central and take full advantage of:}

- Convenient online submission

- Thorough peer review

- No space constraints or color figure charges

- Immediate publication on acceptance

- Inclusion in PubMed, CAS, Scopus and Google Scholar

- Research which is freely available for redistribution

Submit your manuscript at www.biomedcentral.com/submit
C Biomed Central 